\title{
Article \\ Functional and Structural Changes in the Corticospinal Tract of Streptozotocin-Induced Diabetic Rats
}

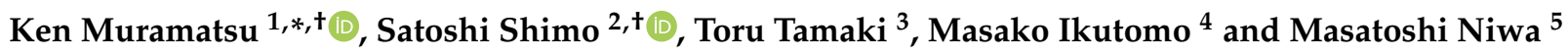 \\ 1 Department of Physical Therapy, Kyorin University, 5-4-1 Simorenzyaku, Mitaka, Tokyo 181-8612, Japan \\ 2 Department of Occupational Therapy, Health Science University, 7187 Kodachi, Fujikawaguchiko, \\ Yamanashi 401-0380, Japan; sshimo@kenkoudai.ac.jp \\ 3 Department of Physical Therapy, Health Science University, 7187 Kodachi, Fujikawaguchiko, \\ Yamanashi 401-0380, Japan; toru-tamaki@kenkoudai.ac.jp \\ 4 Department of Physical Therapy, University of Tokyo Health Sciences, 4-11 Ochiai, Tama, \\ Tokyo 206-0003, Japan; m-ikutomo@u-ths.ac.jp \\ 5 Department of Occupational Therapy, Kyorin University, 5-4-1 Simorenzyaku, Mitaka, Tokyo 181-8612, Japan; \\ mt-niwa@ks.kyorin-u.ac.jp \\ * Correspondence: k-muramatsu@ks.kyorin-u.ac.jp \\ + These authors contributed equally to this manuscript.
}

Citation: Muramatsu, K.; Shimo, S.; Tamaki, T.; Ikutomo, M.; Niwa, M.

Functional and Structural Changes in the Corticospinal Tract of Streptozotocin-Induced Diabetic Rats. Int. J. Mol. Sci. 2021, 22, 10123. http://doi.org/10.3390/ ijms221810123

Academic Editor: Kazunori Sango

Received: 24 August 2021

Accepted: 17 September 2021

Published: 19 September 2021

Publisher's Note: MDPI stays neutral with regard to jurisdictional claims in published maps and institutional affiliations.

Copyright: (c) 2021 by the authors. Licensee MDPI, Basel, Switzerland. This article is an open access article distributed under the terms and conditions of the Creative Commons Attribution (CC BY) license (https:/ / creativecommons.org/licenses/by/ $4.0 /)$.

\begin{abstract}
This study aimed to reveal functional and morphological changes in the corticospinal tract, a pathway shown to be susceptible to diabetes. Type 1 diabetes was induced in 13-week-old male Wistar rats administered streptozotocin. Twenty-three weeks after streptozotocin injection, diabetic animals and age-matched control animals were used to demonstrate the conduction velocity of the corticospinal tract. Other animals were used for morphometric analyses of the base of the dorsal funiculus of the corticospinal tract in the spinal cord using both optical and electron microscopy. The conduction velocity of the corticospinal tract decreased in the lumbar spinal cord in the diabetic animal, although it did not decrease in the cervical spinal cord. Furthermore, atrophy of the fibers of the base of the dorsal funiculus was observed along their entire length, with an increase in the g-ratio in the lumbar spinal cord in the diabetic animal. This study indicates that the corticospinal tract fibers projecting to the lumbar spinal cord experience a decrease in conduction velocity at the lumbar spinal cord of these axons in diabetic animals, likely caused by a combination of axonal atrophy and an increased g-ratio due to thinning of the myelin sheath.
\end{abstract}

Keywords: diabetes; corticospinal tract; diabetic neuropathy

\section{Introduction}

Diabetes patients with neuropathy have shown several motor dysfunctions, including an increased risk of falling, altered gait and balance, and increased body sway [1-4]. These motor dysfunctions were previously believed to be caused by a combination of sensory neuropathy and muscle wasting [5]. However, recent studies have revealed that motor system dysfunction, including the cerebral cortex and spinal cord, can also cause motor dysfunction in these patients [6,7].

We have previously reported disrupted axonal conduction and reductions in the cortical hindlimb motor area of type 1 diabetic rats [8]. Moreover, axonal disruption of the corticospinal tract (CST) is prominent in the long axons that project to the lumbar spinal segment [8]. A previous study reported poor synaptic transmission between the motor cortex and the lumbar spinal motor neurons of short-term type 1 diabetic rats [9], while a clinical study reported a decrease in the volume and conduction velocity of the CST in both type 1 and type 2 diabetes patients [10-15]. Understanding the nature of diabetes-related CST injuries is important for revealing the pathophysiology of motor dysfunction in diabetic patients since this tract plays an essential role in controlling voluntary movements $[6,16]$. However, it is unclear what kind of pathological changes occur in CST 
axons. Therefore, this study investigated the functional and morphological changes in the long axons of the CST that originate from the hindlimb cortical motor area and connect to the lumbosacral segment.

\section{Results}

\subsection{Model Animals}

Diabetic rats exhibited low body weights and blood glucose levels of approximately $400 \mathrm{mg} / \mathrm{dL}(464.5 \pm 43.0 \mathrm{mg} / \mathrm{dL}$ on average, $(p<0.0001) \mathrm{vs.} 23 \mathrm{WC}: 149.7 \pm 15.0 \mathrm{mg} / \mathrm{dL})$ within 2-3 days of streptozotocin (STZ) injection and throughout the study. The control and diabetic animal groups had similar body weights before the STZ or saline injections. However, the body weights of the control group at 23 weeks after sham diabetes induction $(23 \mathrm{WC})$ increased to $449.7 \pm 18.8 \mathrm{~g}$ on average, whereas the body weight of diabetic animals at 23 weeks after diabetes induction (23WD) was $280.4 \pm 6.9 \mathrm{~g}$ on the average $(p<0.0001$, vs. $23 \mathrm{WC})$. The body weights and blood glucose levels of the animals used in each experiment are summarized in Tables 1 and 2.

Table 1. Body weights, blood glucose levels, and conduction velocities of CST fibers in animals used for electrophysiological experiments.

\begin{tabular}{lccc}
\hline & 23WC $(\mathbf{n}=\mathbf{6})$ & 23WD $(\mathbf{n}=\mathbf{7})$ & $p$-Value \\
\hline Body weight $(\mathrm{g})$ & $449.7 \pm 16.6$ & $282.0 \pm 6.0$ & $<0.0001$ \\
Blood sugar (mg/dL) & $152.7 \pm 16.3$ & $465.4 \pm 46.2$ & $<0.0001$ \\
Conduction velocity & & & \\
of the CST (m/s) & & & 0.99 \\
Stimulation: Cervical & $14.7 \pm 1.6$ & $14.3 \pm 1.2$ & 0.02 \\
Stimulation: Lumbar & $16.7 \pm 0.9$ & $12.9 \pm 1.9$ & $<0.01$ \\
MNCVs (m/s) & $56.3 \pm 5.7$ & $39.9 \pm 8.2$ & \\
\hline
\end{tabular}

Abbreviations: 23WC, control animals at 23 weeks after sham diabetes induction; 23WD, diabetic animals at 23 weeks after diabetes induction; CST, corticospinal tract; $\mathrm{MNCV}$, motor nerve conduction velocity.

Table 2. Body weights, blood glucose levels, and morphological data for BDF fibers in animals used for morphometric experiments.

\begin{tabular}{|c|c|c|c|}
\hline & $23 W C(n=4)$ & $23 W D(n=4)$ & $p$-Value \\
\hline Body weight (g) & $449.8 \pm 24.5$ & $277.5 \pm 8.2$ & $<0.0001$ \\
\hline Blood sugar (mg/dL) & $145.3 \pm 13.6$ & $463.1 \pm 45.3$ & $<0.0001$ \\
\hline \multicolumn{4}{|l|}{$\begin{array}{l}\text { Average diameter of } \\
\text { axon }(\mu \mathrm{m})\end{array}$} \\
\hline Cervical & $1.37 \pm 0.11$ & $1.06 \pm 0.07$ & 0.03 \\
\hline Lumbar & $1.07 \pm 0.02$ & $0.94 \pm 0.05$ & 0.03 \\
\hline \multicolumn{4}{|l|}{$\begin{array}{l}\text { Cross-sectional area } \\
\text { of axon }\left(\mu \mathrm{m}^{2}\right)\end{array}$} \\
\hline Cervical & $1.12 \pm 0.20$ & $0.90 \pm 0.09$ & 0.06 \\
\hline Lumbar & $1.04 \pm 0.04$ & $0.78 \pm 0.11$ & 0.03 \\
\hline \multicolumn{4}{|l|}{$\begin{array}{l}\text { Perimeter of axon } \\
(\mu \mathrm{m})\end{array}$} \\
\hline Cervical & $4.38 \pm 0.51$ & $3.78 \pm 0.26$ & 0.06 \\
\hline Lumbar & $3.96 \pm 0.20$ & $3.31 \pm 0.28$ & 0.03 \\
\hline \multicolumn{4}{|l|}{ G-ratio } \\
\hline Cervical & $0.65 \pm 0.03$ & $0.66 \pm 0.01$ & 0.69 \\
\hline Lumbar & $0.62 \pm 0.05$ & $0.72 \pm 0.03$ & 0.03 \\
\hline \multicolumn{4}{|l|}{$\begin{array}{l}\text { Myelin thickness } \\
(\mu \mathrm{m})\end{array}$} \\
\hline Cervical & $0.54 \pm 0.05$ & $0.38 \pm 0.01$ & 0.03 \\
\hline Lumbar & $0.42 \pm 0.03$ & $0.33 \pm 0.04$ & 0.03 \\
\hline
\end{tabular}

Abbreviations: BDF, base of the dorsal funiculus; 23WC, control animals at 23 weeks after sham diabetes induction; $23 \mathrm{WD}$, diabetic animals at 23 weeks after diabetes induction; CST, corticospinal tract. 


\subsection{Conduction Velocities of the CST and Motor Nerve}

The antidromic field potential of the CST neurons was recorded from the hindlimb cortical motor area. The conduction velocity was then calculated from the shortest latency of the antidromic field potentials, which did not differ between 23WC and 23WD when stimulating the CST at the cervical spinal level (Figure 1a,b, Table 1). However, 23WD showed long-lasting potentials that followed the first sharp potential and frequently merged with the synaptic potential of CST neurons. Therefore, a quantitative analysis of these late potentials was not performed. Meanwhile, the latency for the antidromic field potential of CST neurons of 23WD was prolonged when stimulating the lumbar spinal level. The conduction velocity decreased to $77.2 \%$ of $23 W C(p<0.05$, Figure $1 \mathrm{c}, \mathrm{d}$, Table 1$)$. The potentials that followed the first sharp potentials were longer than those with cervical stimulation. Multiple antidromic field potentials with different conduction velocities were recorded in a few cases when stimulating the spinal cord at supra-maximal strength. Figure 2 shows a typical record in which the shortest latency potential is followed by an exceedingly long latency potential. The potentials with the longest latencies sometimes reached $15-20 \mathrm{~ms}$, corresponding to conduction velocities of $2-3 \mathrm{~m} / \mathrm{s}$. These data were not included in the analysis because they were unstable, and the extent of the stimulating current effect could not be estimated. Lastly, the motor nerve conduction velocities (MNCVs) were significantly decreased in $23 W D(70.9 \%$ of $23 W C, p<0.001)$.

a

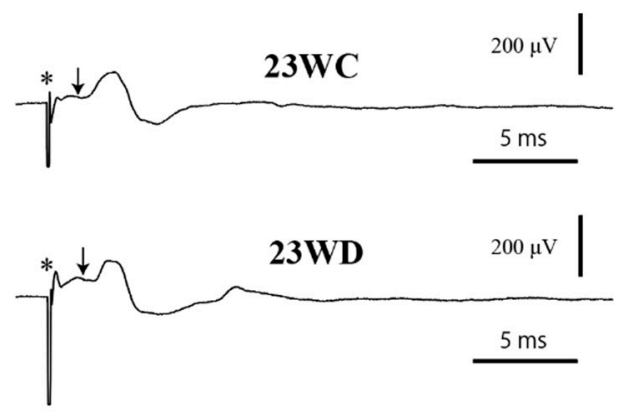

c

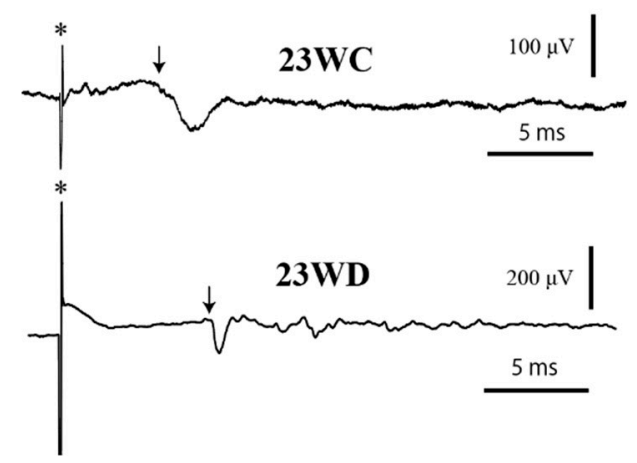

b

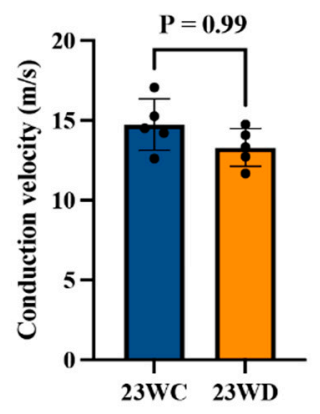

d

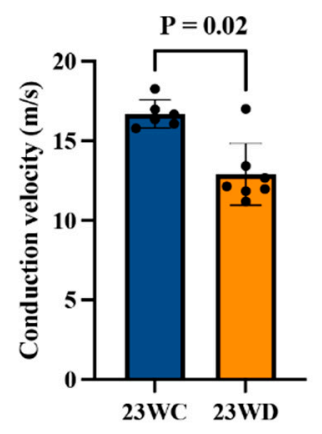

Figure 1. Typical evoked antidromic field potentials of corticospinal tract (CST) neurons. (a) Typical hindlimb-area antidromic potentials evoked by C3-segment stimulation in 23WC and 23WD animals. (b) There was no significant difference in conduction velocity between diabetic animals and control animals. On the other hand, 23WD showed long-lasting potentials. (c) Typical hindlimb-area antidromic potentials evoked by L1-segment stimulation in 23WC and 23WD animals. (d) The diabetic animals exhibited decreased conduction velocities and long-lasting multiphasic potentials. Asterisks indicate stimulus artifacts, and arrows indicate each action potential's first negative deflection. Abbreviations: $23 \mathrm{WC}$, control animals at 23 weeks after sham diabetes induction; $23 \mathrm{WD}$, diabetic animals at 23 weeks after diabetes induction. 


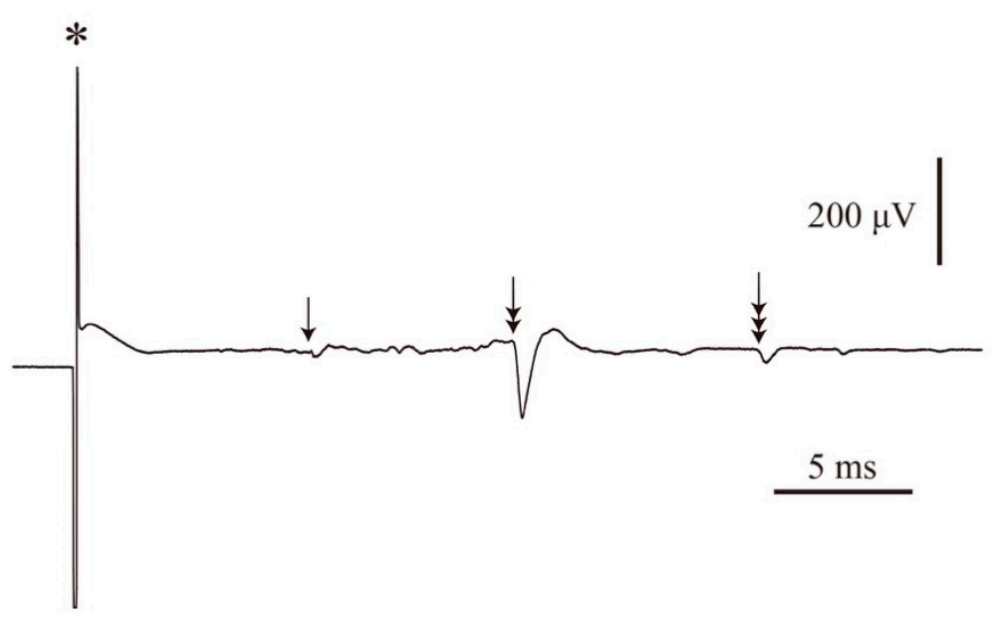

Figure 2. Multiple field potentials of the corticospinal tract (CST) with different conduction velocities. We occasionally observed multiple field potentials in CST neurons with different conduction velocities when applying supra-maximal stimulation to the spinal cord. In this case, the potential with the shortest latency (arrow) is followed by potential with a long latency (double arrow) and then by potential with an extremely long latency (triple arrow). In this case, the conduction velocity of the potential with the longest latency was about $3 \mathrm{~m} / \mathrm{s}$. Asterisks indicate stimulus artifacts.

\subsection{Morphological Analysis of Tract Fibers at the Base of the Dorsal Funiculus}

Detailed figures for each value are summarized in Table 2. Figure 3a,d, and Figure 4a,d show tissue images from the base of the dorsal funiculus (BDF) of the cervical and lumbar spinal cords occupied by CST fibers $[17,18]$. As shown in Figure 3b,e,g and Figure 4b,e,g, the myelinated fibers in the dorsal funiculus were generally thinner in $23 \mathrm{WD}$, and the average diameter of the BDF axons was smaller than that of 23WC $(p<0.05)$. The histogram shows an increase in the ratio of thin BDF fibers due to axonal atrophy in the 23WD group, and the distribution was different from that in the 23WC group (Figures $3 \mathrm{~h}$ and $4 \mathrm{~h}$, Kolmogorov-Smirnov test, $p<0.0001$ ).

There were some differences between the cervical and lumbar spinal cords in other measures reflecting the size of long tract axons. In the cervical spinal segment, the crosssectional area and perimeter of the axons showed only a tendency to be decreased in the $23 W D$ group $(p=0.06)$, while, in the lumbar spinal segment, the cross-sectional area and perimeter of axons in the 23WD group were significantly smaller than those of the 23WC group $(p<0.05)$. In addition to axonal atrophy, the myelin thickness of 23WD reduced in both the cervical and lumbar segments compared to 23WC. The g-ratios were similar (approximately 0.65) at the cervical and lumbar segments of 23WC and the cervical segment of 23WD (Figure 3i,j); however, the g-ratio of the lumbar segment of 23WD showed an increased mean value $(0.72 \pm 0.03, p<0.05$, Figure $4 \mathrm{i})$. As shown in Figure $4 \mathrm{j}$, the increase in the g-ratio at the lumbar spinal cord did not occur uniformly in all long tract fibers. Rather, it was caused by a substantial increase in the g-ratio of some fibers.

The electro-microscopic images reflected this trend more clearly. At the cervical spinal cord level, BDF fibers appeared to have preservation of myelinated structures, although thin axons were evident (Figure $3 b, c, e, f$ ). On the other hand, BDF fibers of the lumbar spinal cord were thin and had wide fiber spacing in 23WD, with some fibers having thin myelin sheaths (Figure $4 b, c, e, f)$. Structurally disrupted fibers, i.e., demyelinated or degenerated axons, were rarely observed in the cervical and lumbar spinal cord. 

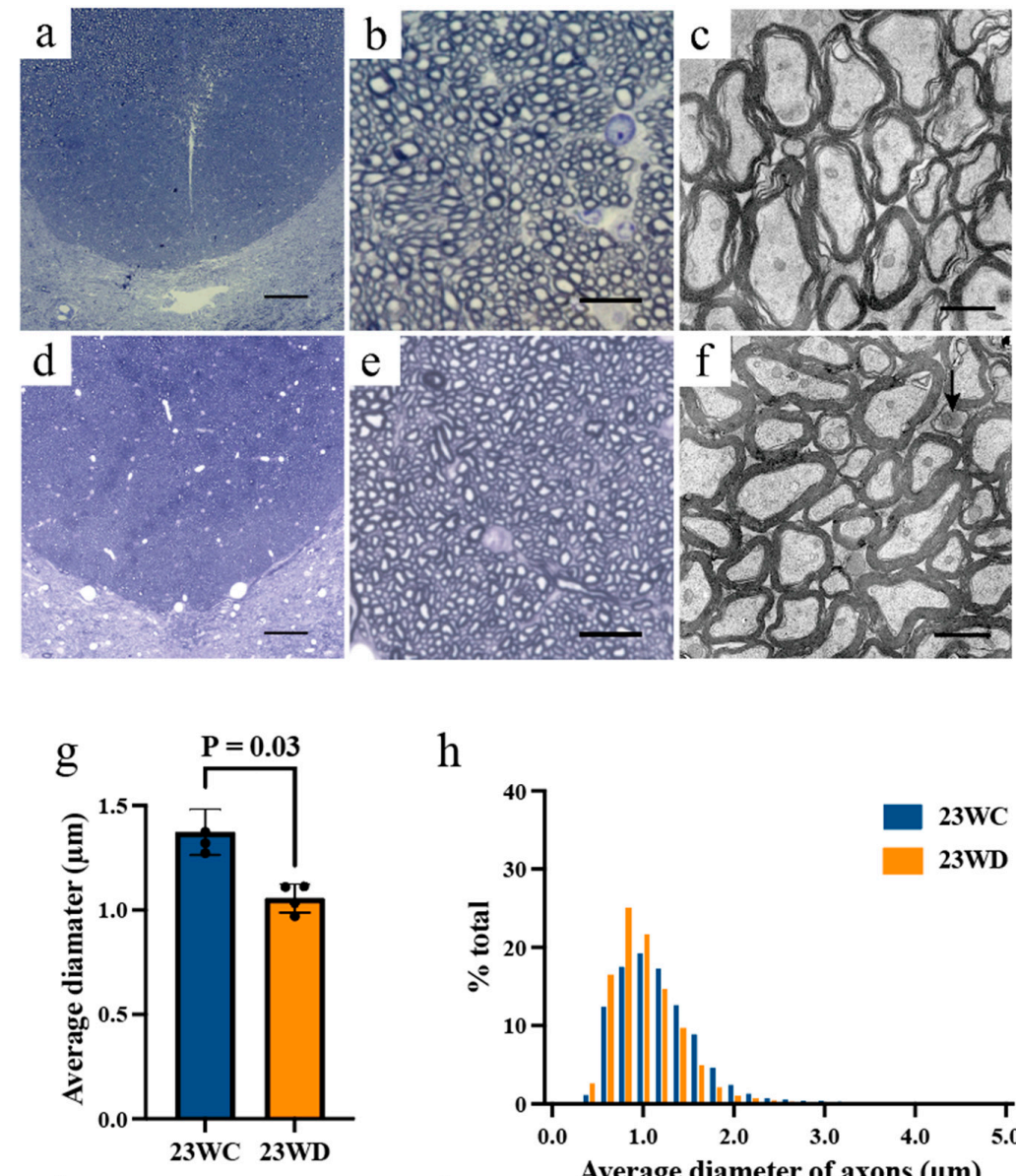

h

i
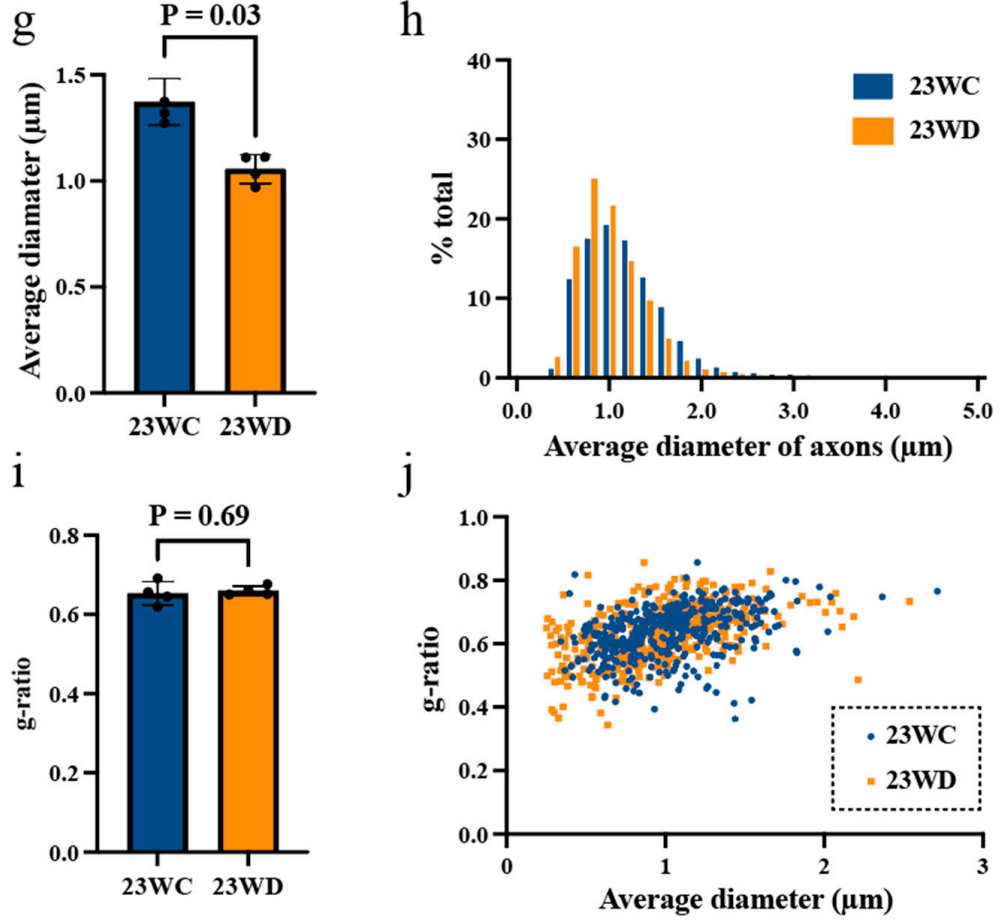

Figure 3. (a,b) Optical micrographs of the BDF in the cervical spinal cord (a) and BDF fibers (b) in 23WC. (c) Electron micrographs of BDF fibers in 23WC. (d,e) Optical micrographs of the BDF of the cervical spinal cord (d) and BDF fibers (e) in 23WD. Evidently, the BDF fibers are thinner in 23WD than 23WC. (f) Electron micrographs of BDF fibers in 23WD. The arrow indicates a degenerating axon. No changes in the myelin sheath were observed, and degenerating axons were only rarely observed. The scale bar indicates $100 \mu \mathrm{m}(\mathbf{a}, \mathbf{d}), 10 \mu \mathrm{m}(\mathbf{b}, \mathbf{e})$, and $1 \mu \mathrm{m}(\mathbf{c}, \mathbf{f})$. (g) Average diameter of BDF axons. The size of axons was significantly smaller in 23WD $(p<0.05$, Mann-Whitney U test). (h) Size distribution of BDF axons in the cervical spinal cord in 23WC ( $\mathrm{n}=12,256$ axons) and 23WD ( $n=10,673$ axons). The Kolmogorov-Smirnov test revealed a significant $(p<0.0001)$ between-group difference. (i) g-ratio of BDF fibers. There was no significant difference between the two groups $(p<0.05$, Mann-Whitney $\mathrm{U}$ test). (j) The relationship between axonal diameter and the g-ratio in 23WC ( $n=420$ fibers) and 23WD ( $n=431$ fibers). There was no difference between the two groups. Abbreviations: BDF, base of the dorsal funiculus; 23WC, control animals at 23 weeks after sham diabetes induction; 23WD, diabetic animals at 23 weeks after diabetes induction. 

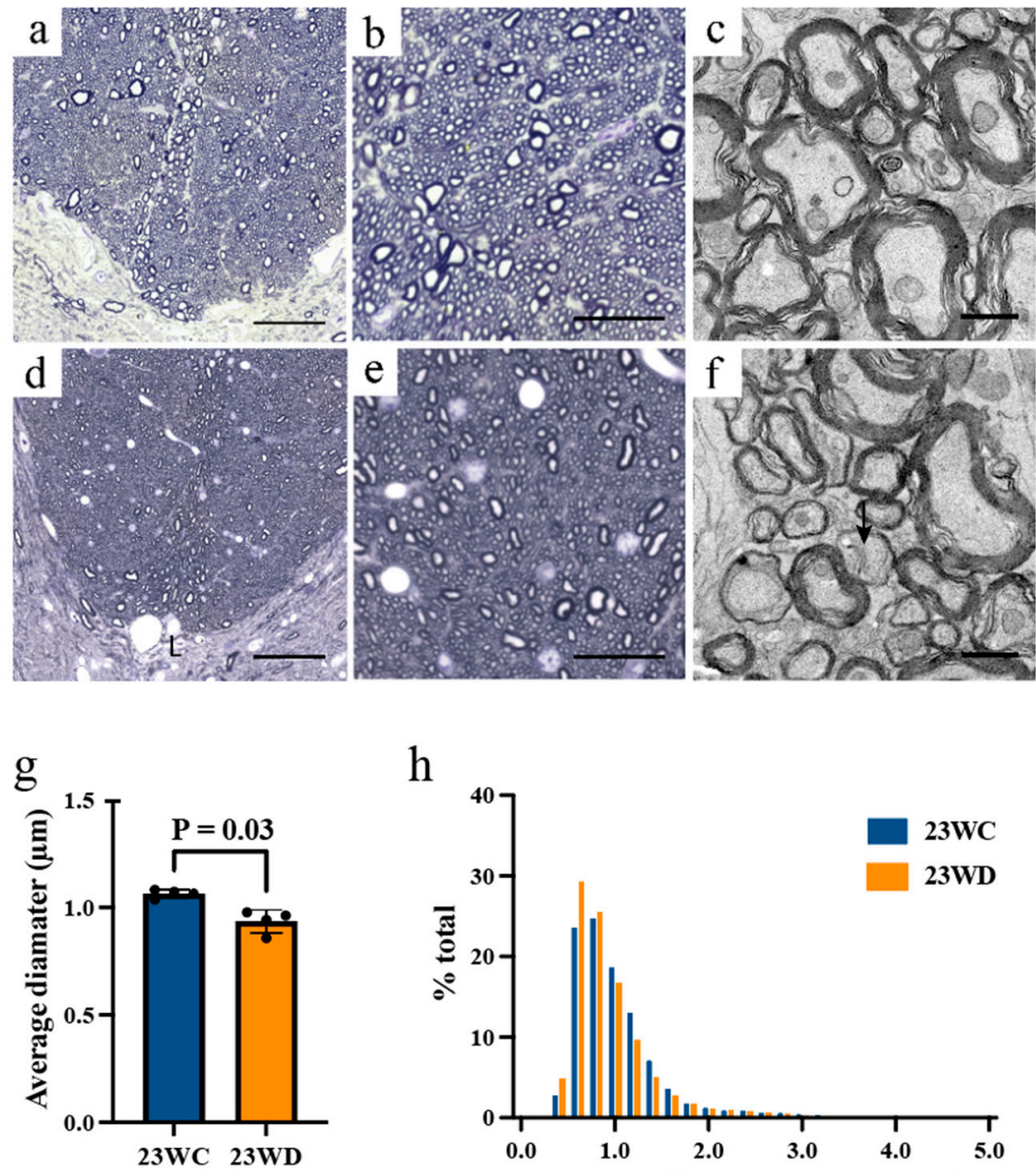

h

i
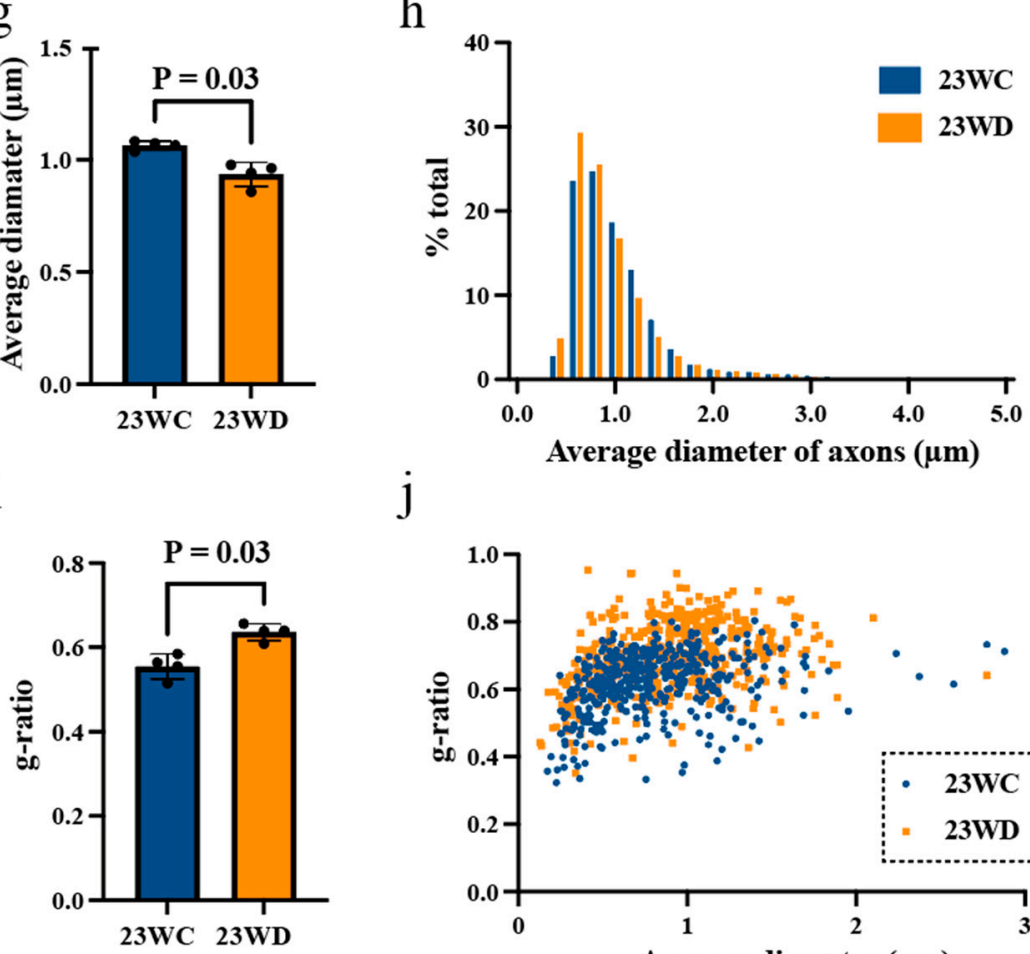

Average diameter of axons $(\mu \mathrm{m})$

j

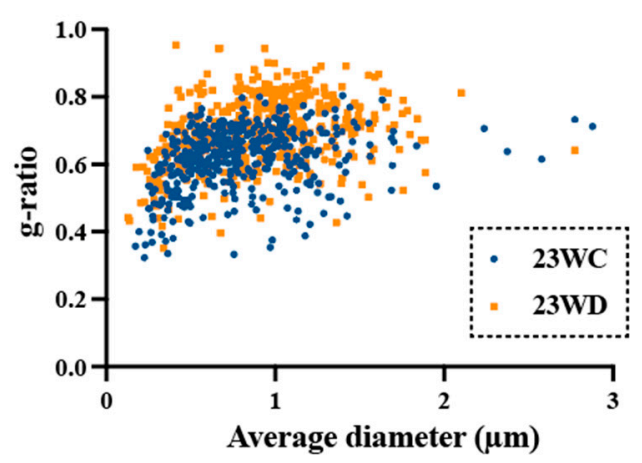

Figure 4. (a,b) Optical micrographs of the BDF of the lumbar spinal cord (a) and BDF fibers (b) in 23WC. (c) Electron micrographs of BDF fibers in 23WC. (d,e) Optical micrographs of the bottom of the dorsal column of the cervical spinal cord (d) and BDF fibers (e) in 23WD. (f) Electron micrographs of BDF fibers in 23WD. The arrow indicates a demyelinating axon. The scale bar indicates $100 \mu \mathrm{m}$ $(\mathbf{a}, \mathbf{d}), 10 \mu \mathrm{m}(\mathbf{b}, \mathbf{e})$, and $1 \mu \mathrm{m}(\mathbf{c}, \mathbf{f})$. (g) Average diameter of BDF axons. The axons were significantly smaller in 23WD ( $p<0.05$, Mann-Whitney U test). (h) Size distribution of BDF axons in the lumbar cord in 23WC ( $\mathrm{n}=10,220$ axons) and 23WD ( $\mathrm{n}=9630$ axons). The Kolmogorov-Smirnov test revealed a significant $(p<0.0001)$ between-group difference. (i) g-ratio of BDF fibers. In 23WD, the g-ratio was significantly higher than in 23WC ( $p<0.05$, Mann-Whitney $\mathrm{U}$ test). ( $\mathrm{j}$ ) The relationship between axonal diameter and the g-ratio in 23WC ( $\mathrm{n}=439$ fibers) and 23WD ( $\mathrm{n}=438$ fibers). In 23WD, the g-ratio of some BDF fibers increased independent of axonal diameter. Abbreviations: BDF, base of the dorsal funiculus; $23 \mathrm{WC}$, control animals at 23 weeks after sham diabetes induction; 23WD, diabetic animals at 23 weeks after diabetes induction. 


\section{Discussion}

The study found that the axons at the lumbar spinal level developed functional disorders, such as a decreased conduction velocity and morphological abnormalities, such as axonal atrophy and an increased g-ratio. These findings are consistent with the results of our previous study, which demonstrated that diabetes-induced cortical motor area reductions predominantly affected the hindlimb and trunk areas [8].

Electro-physiological analysis revealed that the conduction velocity of CST fibers decreased in 23WD. Because antidromic field potentials from the hindlimb motor area were recorded, these alterations reflected the differences in the effects of diabetes at different portions of the CST fibers that connect to the lumbar spinal level. A comparison between the conduction velocities of stimulated CST fibers at the cervical and lumbar levels revealed that the decrease in conduction velocity only occurred at the lumbar level (Figure 1). This is similar to the finding that peripheral motor nerves have a predominantly distal decrease in conduction velocity in diabetes [19]. Conversely, it was previously reported that the conduction velocity of the CST originating from the forelimb motor cortices and projecting to the cervical spinal cord shows a decreased conduction velocity in 23-week diabetic rats [8]. At first glance, these findings seem to contradict one another. However, it is believed that this discrepancy is because the CST fibers originating from the forelimb area stimulate the terminal portion of the fibers, while the CST fibers originating from the hindlimb region stimulate the central portion of the fibers. The conduction velocity of CST axons calculated by the shortest latency of the antidromic field potential should be noted. This reflects the conduction velocity of the fastest, i.e., the largest, CST axons. A previous study showed that the CST contains fibers of various sizes and conduction velocities; therefore, analyzing only the fastest conduction velocity does not provide a complete picture of its reduction in the CST [20]. The inability to present sufficient data to back up this point is a limitation of this study's methodology. However, as shown in Figure 2, longlatency antidromic field potentials of CST cells with slow conduction velocities in 23WD were occasionally recorded. Based on these observations, it is believed that the decrease in conduction velocity also occurred in smaller CST fibers with a slower conduction velocity because these potentials were only observed by supra-maximal stimulation, and the conduction velocities of these CST fibers included some as low as $2-3 \mathrm{~m} / \mathrm{s}$, which is below the minimum velocity of CST fibers in normal rats (approximately $4 \mathrm{~m} / \mathrm{s}$ ) [20]. This decrease in the nerve conduction velocity may significantly affect the arrival time of motor commands from the cortical motor cortex to the spinal cord. Since the effect of a reduced conduction velocity is greater, the longer the distance from the excitation, the more time for motor commands to arrive at the lumbosacral spinal cord, which is farthest from the cortical motor cortex, may be completely different for each CST fiber. This may make it difficult to supply synchronized excitatory synaptic input to hindlimb motor neurons and provide a good explanation for why diabetes-induced cortical motor area reductions predominantly affect the hindlimb and trunk areas [8].

The morphological observations also reflected these physiological explanations. The morphological analysis focused on the alterations at the BDF, where the CST is located. Since CST axons were not identified using a neurotracer, other axons may also have been included in the axons analyzed. However, it has previously been established that CST axons occupy the BDF of rats and fully reflect the morphological features of CST axons $[17,18]$. Therefore, the fibers at the BDF are referred to as putative CST (p-CST) fibers for convenience in the discussion. In addition, the CST at the cervical spinal cord contains fibers that project to segments other than the lumbar spinal cord. Since these could not be distinguished in this study, it is another limitation of our experimental methodology.

The quantitative morphological analysis of p-CST fibers revealed that atrophy of axons was diffused over the total length of the p-CST but was most evident in the lumbar spinal segment of 23WD. In addition to morphological changes in axons, a decrease in p-CST fibers myelin thickness was also observed in the cervical and lumbar segments of $23 \mathrm{WD}$. It is widely accepted that the g-ratio is a highly reliable ratio for assessing axonal 
myelination [21]. Furthermore, it is known that the g-ratio of the CST in young rats is approximately 0.65 , which is fairly constant throughout the diameter spectrum $[17,18]$. As shown in Figure 3i,j, the g-ratio of 23WC showed similar values at the cervical and lumbar segments, as well as the cervical segment of $23 \mathrm{WD}$. However, the g-ratio of the lumbar segment of $23 \mathrm{WD}$ showed an increased mean value $(0.72 \pm 0.03$, see Figure $4 \mathrm{i}$ and Table 2$)$. It is believed that a g-ratio of 0.7 in the CNS does not cause interference with excitation conduction [21]. However, Figure 4j, shows the increase in the lumbar spinal cord g-ratio did not occur uniformly for all p-CST fibers but was caused by a large increase in the g-ratio of over 0.7 in some $\mathrm{p}$-CST fibers. Therefore, $\mathrm{p}$-CST fibers in lumbar spinal segments with significantly increased g-ratios may substantially impair excitatory conduction when coupled with axonal atrophy since it is known that both the axonal diameter and myelin sheath are important for minimizing conduction delays [21]. This observation provides a good explanation for our electrophysiological data, which demonstrated a decrease in the conduction velocity predominantly in the lumbar spinal segment of p-CST fibers in 23WD. However, why the myelin abnormalities of p-CST axons are more prominent in the lumbar spinal segment is unclear. This could be due to the various known interactions between oligodendrocytes and axons that may influence myelination [22]. Another possibility is that CST fibers degenerate secondary to hindlimb motor neuron damage because MNCVs decreased in 23WD. However, this is unlikely, because previous studies have shown motor neuron damage in the same animal model has little effect on alpha motor neurons $[23,24]$. On the other hand, it has been previously predicted that axonal retraction contributes to reducing cortical motor area [8]. However, functional alterations in p-CST fibers caused by axonal atrophy and demyelination, rather than axonal retraction, seem to account for most of the changes in the motor cortex because the histological analysis showed that axonal retraction was rarely observed.

In conclusion, defects in the corticospinal tract observed in STZ rats are characterized by axonal atrophy and thinning of the myelinated sheaths in peripheral regions, as well as a decreased nerve conduction velocity and an increased conduction velocity variability, probably due to the aforementioned changes. This decreased conduction velocity and increased variability may explain why synchronized synaptic inputs to the spinal cord are prevented, resulting in the failure of excitatory transmission from the cerebral cortex to MNs. However, limitations of this study include the following: first, it did not measure the conduction velocity of axons in a single cell; therefore, it cannot clarify the extent to which the conduction velocity varied. Second, it did not identify CST fibers using neurotracers and others. A method to visualize entire CST fibers without affecting their morphology needs to be investigated. Third, the 3D ultrastructural architecture of unmyelinated axons, axoplasm, or myelin sheaths of myelinated axons was not observed, making it impossible to investigate the changes within the cells. If the conduction velocity of axons in a single cell could be observed and quantify 3D ultrastructure of CST fibers, a new mechanism of pathogenesis could be found, which should be studied in future.

\section{Materials and Methods}

\subsection{Induction of Experimental Diabetes (Diabetic Animal Model)}

Type 1 diabetes was induced in 13-week-old male Wistar rats $(n=11), 270-288 \mathrm{~g}$ in body weight, via STZ (100 mg/ $\mathrm{kg}$ in saline, i.p.) administration, and a plasma glucose level of $>400 \mathrm{mg} / \mathrm{dL}$ was used to confirm a diabetic status. Age-matched control animals (268-277 g body weight) were injected with saline only $(n=10)$. All animals were housed in plastic cages with a flat bottom covered with a soft bedding material. Food and tap water were provided ad libitum. Animals were maintained in a temperature-controlled room with a light/dark cycle of 12:12 h. Twenty-three weeks after STZ injection-determined based on a previous study demonstrating that diabetes induced during this period caused the most obvious CST injury-the diabetic group $(23 \mathrm{WD}, \mathrm{n}=7)$ and control group (23WC, $\mathrm{n}=6$ ) were used to demonstrate the conduction velocity of the CST [8]. The third group was used for ultrastructural and morphometric analyses (each group containing four animals). 


\subsection{Measurement of the Conduction Velocity of CST Fibers and Motor Nerve}

23WD and 23WC rats were used to demonstrate the conduction velocity of CST fibers, as previously described [8]. In brief, animals were anesthetized with ketamine hydrochloride $(70 \mathrm{mg} / \mathrm{kg}$, i.p.) and xylazine $(5 \mathrm{mg} / \mathrm{kg}$, i.p.), then were mounted in a stereotaxic frame and placed on a heating pad. A rectal thermometer was used to confirm a body temperature of $37^{\circ} \mathrm{C}$. Craniotomies were performed to expose the right sensorimotor cortex, and 1.4-M $\Omega$ tungsten recording microelectrodes were inserted at the center of the hindlimb motor area. Laminectomies was performed to expose the dorsal surface of the spinal cord at the C3 and L1 segments to stimulate the CST at the spinal level. Subsequently, $40-\mathrm{k} \Omega$ tungsten microelectrodes was stereotaxically inserted (Unique Medical, Tokyo, Japan) at the deepest portion of the left dorsal column where the rat CST was located [17].

To record the antidromic potentials of CST neurons, the spinal cord was stimulated with single-pulse, $1-\mathrm{Hz}, 200-\mu \mathrm{s}$ monophasic cathodal pulses from SEN-7103 stimulators (Nihon Kohden, Tokyo, Japan) and SS-04 J isolators (Nihon Kohden, Tokyo, Japan). The maximum stimulation current was $50 \mu \mathrm{A}$ to avoid stimulation of other pathways by leaked current pathways. In five animals per group, both the cervical and lumbar spinal segments were stimulated while stimulating only the lumbar segment in the reining animals. The stimulating current was gradually increased until antidromic field potentials were recorded. These potentials were amplified (AB-651 J, Nihon Kohden) and digitized at a 20-kHz sample rate (PowerLab, ADInstruments, Dunedin, New Zealand), and blocks of 10 consecutive potentials were averaged (Chart 8, ADInstruments, Dunedin, New Zealand). A potential with a latency that fluctuated between sweeps was frequently observed. However, these potentials were excluded from analyses because they might have resulted from synaptically activated neurons by somatosensory cortex rather than antidromically activated neurons [20]. The distance from the stimulation point to each recording point was determined at the end of each experiment. The latency of potentials was calculated from the onset time of the stimulus artifact to the point of the first deflection of the evoked potential in the averaged recording. The CST conduction velocities were calculated.

Subsequently, we measured the peripheral MNCVs, exposed the right sciatic nerve and sectioned it at the ischiorectal fossa. The nerves on either side of the cut were mounted on a bipolar silver hook electrode and inserted bipolar wire electrodes into the medial gastrocnemius muscle to record evoked electromyograms. The average MNCVs was determined by stimulating nerves with $2-\mathrm{Hz}, 100-\mu$ s supra-maximal pulse stimuli. We averaged 10 responses and then calculated the MNCVs by subtracting the distal latency from the proximal latency and dividing the result by the inter-electrode distance.

\subsection{Morphological Analysis}

Anesthesia was initially induced with $4 \%$ halothane and then subsequently administered pentobarbital $(35 \mathrm{mg} / \mathrm{kg}$, i.p.) and transcardially perfused animals with $500 \mathrm{~mL}$ of saline containing heparin sodium followed by a fixative solution of $4 \%$ paraformaldehyde and $1 \%$ glutaraldehyde in a $0.1-\mathrm{M}$ phosphate buffer $(\mathrm{pH} 7.4)$ at $4{ }^{\circ} \mathrm{C}$. The spinal cords were removed and placed in the same solution at $4{ }^{\circ} \mathrm{C}$ for $24-48 \mathrm{~h}$, as reported previously [25]. The spinal cords were then sectioned transversely into 1-mm blocks, post-fixed with $1 \%$ osmium tetroxide in $0.1 \mathrm{M} \mathrm{PB}$, dehydrated in a graded series of ethanol, and routinely embedded in Epon. The Epon-embedded specimens were first cut into $1.0-\mu \mathrm{m}$ thick sections with an ultramicrotome, mounted on glass slides (Matsunami Glass, Osaka, Japan), and routinely stained with toluidine blue to identify surface tissue areas under a BZ-X710 multifocal microscope (KEYENCE, Osaka, Japan) for examination and photographing of the dorsal column of the spinal cord. Ultrathin sections were then cut at a 70-90 nm thickness adjacent to the thicker Epon sections. These were doubly stained with uranyl acetate and lead citrate and then observed and photographed using a transmission electron microscope (JEM-2100F, JEOL, Tokyo, Japan) at an accelerating voltage of $200 \mathrm{kV}$.

Subsequently, the morphology, axonal size, myelin thickness, and g-ratio (the ratio of the inner axonal diameter to the total outer diameter) of the myelinated fibers located 
$200 \mu \mathrm{m}$ from the BDF were analyzed, which is occupied by CST fibers in photographs with Image (National Institutes of Health, Bethesda, MD) [17,18]. The sizes of the axons were measured using optical micrographs. An imaginary oval surrounding the fiber or axon was used for the diameter measurements. The myelin thickness and g-ratio were calculated from a random selection of more than 100 fibers from each group using a combination of optical and electron micrographs because the boundaries between adjacent fibers could be obscured. Although there were larger myelinated fibers scattered in the center of the base of the dorsal column, these were fibers of the ascending tract and were therefore excluded from the analysis [26].

\subsection{Data Analysis}

The data are presented as means \pm standard deviations. Between-group comparisons were performed using the Mann-Whitney U test. The Kolmogorov-Smirnov 2-sample test was used to determine whether the distributions were significantly different. All analyses were performed using GraphPad Prism 9 software (GraphPad Software, La Jolla, CA, USA). Statistical significance was defined as a $p$-value of $<0.05$, with a tendency toward significance defined as $0.05 \leq p<0.15$.

Author Contributions: Conceptualization, K.M. and S.S.; methodology, K.M. and S.S.; investigation, K.M., S.S., T.T. and M.I.; writing-original draft preparation, K.M. and S.S.; writing-review and editing, M.N.; visualization, M.I.; supervision, M.N.; project administration, K.M.; funding acquisition, K.M. All authors have read and agreed to the published version of the manuscript.

Funding: This research was funded by the Japan Society for the Promotion of Science (KAKENHI) (grant number 18K10726).

Institutional Review Board Statement: All animal experiments were conducted in accordance with the National Institutes of Health Guide for the Care and Use of Laboratory Animals (NIH Publications No. 8023, revised 1978). Animal experimental protocols were approved by the Kyorin University Animal Ethics Committee (No. I18-12, 1 April 2018).

Acknowledgments: The authors thank Masue Muramatsu for help with discussions and Fumio Muramatsu for advice on preparing tissue samples.

Conflicts of Interest: The authors declare no conflict of interest.

\section{References}

1. Maurer, M.S.; Burcham, J.; Cheng, H. Diabetes mellitus is associated with an increased risk of falls in elderly residents of a long-term care facility. J. Gerontol. A Biol. Sci. Med. Sci. 2005, 60, 1157-1162. [CrossRef] [PubMed]

2. Ijzerman, T.H.; Schaper, N.C.; Melai, T.; Meijie, K.; Willems, P.J.B.; Savelberg, H.H.C.M. Lower extremity muscle strength is reduced in people with type 2 diabetes, with and without polyneuropathy, and is associated with impaired mobility and reduced quality of life. Diabetes Res. Clin. Pract. 2012, 95, 345-351. [CrossRef] [PubMed]

3. Allet, L.; Armand, S.; Golay, A.; Monnin, D.; Bie, R.A.; Burin, E.D. Gait characteristics of diabetic patients: A systematic review. Diabetes Metab. Res. Reve. 2008, 24, 173-191. [CrossRef] [PubMed]

4. Brown, S.J.; Handsaker, J.C.; Bowling, F.L.; Boulton, A.J.M.; Reeves, N.D. Diabetic peripheral neuropathy compromises balance during daily activities. Diabetes Care 2015, 38, 1116-1122. [CrossRef]

5. Andersen, H. Motor function in diabetic neuropathy. Acta Neurol. Scand. 1999, 100, 211-220. [CrossRef]

6. Ferris, J.K.; Inglis, J.T.; Madden, K.M.; Boyd, L.A. Brain and Body: A Review of Central Nervous System Contributions to Movement Impairments in Diabetes. Diabetes 2020, 69, 3-11. [CrossRef]

7. Muramatsu, K. Diabetes Mellitus-Related Dysfunction of the Motor System. Int. J. Mol. Sci. 2020, 21, 7485. [CrossRef]

8. Muramatsu, K.; Ikutomo, M.; Tamaki, T.; Shimo, S.; Niwa, M. Effect of streptozotocin-induced diabetes on motor representations in the motor cortex and corticospinal tract in rats. Brain Res. 2018, 1680, 115-126. [CrossRef]

9. Madsen, J.G.; Østergaard, J.A.; Andersen, H.; Pedersen, M. Attenuation of Cortically Evoked Motor-Neuron Potential in Streptozotocin-Induced Diabetic Rats: A Study about the Effect of Diabetes upon Cortical-Initiated Movement. Biomed. Res. Int. 2020, 2020, 1942534. [CrossRef]

10. van Duinkerken, E.; Schoonheim, M.M.; IJzerman, R.G.; Klein, M.; Ryan, C.M.; Moll, A.C.; Snoek, F.J.; Barkhof, F.; Diamant, M.; Pouwels, P.J.W. Diffusion tensor imaging in type 1 diabetes: Decreased white matter integrity relates to cognitive functions. Diabetologia 2012, 55, 1218-1220. [CrossRef] 
11. Xiong, Y.; Sui, Y.; Xu, Z.; Zhang, Q.; Karaman, M.M.; Cai, K.; Anderson, T.M.; Zhu, W.; Wang, J.; Zhou, X.J. Diffusion Tensor Imaging Study on White Matter Abnormalities in Patients with Type 2 Diabetes Using Tract-Based Spatial Statistics. AJNR Am. J. Neuroradiol. 2016, 37, 1462-1469. [CrossRef]

12. Van Bloemendaal, L.; Ijzerman, R.G.; Jennifer, S.; Barkhof, F.; Diamant, M.; Veltman, D.J.; van Duinkerken, E. Alterations in white matter volume and integrity in obesity and type 2 diabetes. Metab. Brain Dis. 2016, 31, 621-629. [CrossRef]

13. Abbruzzese, G.; Schenone, A.; Scramuzza, G.; Caponnetto, C.; Gasparetto, B.; Adezati, L.; Abbruzzese, M.; Viviani, G.L. Impairment of central motor conduction in diabetic patients. Electroencephalogr. Clin. Neurophysiol. 1993, 89, 335-340. [CrossRef]

14. Dolu, H.; Ulas, U.H.; Bolu, E.; Ozkardes, A.; Odabasi, Z.; Ozata, M.; Vural, O. Evaluation of central neuropathy in type II diabetes mellitus by multimodal evoked potentials. Acta Neurologica Belgica 2003, 103, 206-211. [PubMed]

15. Goldenberg, Z.; Kucera, P.; Brezinova, M.; Kurca, E.; Barak, L.; Traubner, P. Clinically unapparent central motor pathways lesion in patients with type I diabetes mellitus. A transcranial magnetic stimulation study. Bratisl Lek Listy 2004, 105, 400-403. [PubMed]

16. Lemon, R.N. Descending pathways in motor control. Annu. Rev. Neurosci. 2008, 31, 195-218. [CrossRef]

17. Saliani, A.; Perraud, B.; Duval, T.; Stikov, N.; Rossignol, S.; Cohen-Adad, J. Axon and Myelin Morphology in Animal and Human Spinal Cord. Front. Neuroanat. 2017, 11, 129. [CrossRef] [PubMed]

18. Brösamle, C.; SAMLE, C.; Schwab, M.E. Cells of origin, course, and termination patterns of the ventral, uncrossed component of the mature rat corticospinal tract. J. Comp. Neurol. 1997, 386, 293-303. [CrossRef]

19. Kimura, J.; Yamada, T.; Nelson, P.S. Distal slowing of motor nerve conduction velocity in diabetic polyneuropathy. J. Neurol. Sci. 1979, 42, 291-302. [CrossRef]

20. Mediratta, N.K.; Nicoll, J.A. Conduction velocities of corticospinal axons in the rat studied by recording cortical antidromic responses. J. Physiol. 1983, 336, 545-561. [CrossRef]

21. Chomiak, T.; Hu, B. What is the optimal value of the g-ratio for myelinated fibers in the rat CNS? A theoretical approach. PLoS ONE 2009, 4, e7754. [CrossRef] [PubMed]

22. Foster, A.Y.; Bujalka, H.; Emery, B. Axoglial interactions in myelin plasticity: Evaluating the relationship between neuronal activity and oligodendrocyte dynamics. Glia 2019, 67, 2038-2049. [CrossRef]

23. Muramatsu, K.; Niwa, M.; Nagai, M.; Kamimura, T.; Sasaki, S.-I.; Ishiguro, T. The size of motoneurons of the gastrocnemius muscle in rats with diabetes. Neurosci. Lett. 2012, 531, 109-113. [CrossRef] [PubMed]

24. Muramatsu, K.; Niwa, M.; Tamaki, T.; Ikutomo, M.; Masu, Y.; Hasegawa, T.; Shimo, S.; Sasaki, S.-I. Effect of streptozotocin-induced diabetes on motoneurons and muscle spindles in rats. Neurosci. Res. 2017, 115, 21-28. [CrossRef] [PubMed]

25. Sathornsumetee, S.; McGavern, D.B.; Ure, D.R.; Rodriguez, M. Quantitative ultrastructural analysis of a single spinal cord demyelinated lesion predicts total lesion load, axonal loss, and neurological dysfunction in a murine model of multiple sclerosis. Am. J. Pathol. 2000, 157, 1365-1376. [CrossRef]

26. Smith, K.J.; Bennett, B.J. Topographic and quantitative description of rat dorsal column fibres arising from the lumbar dorsal roots. J. Anat. 1987, 153, 203-215. 